\title{
A NEW METHOD FOR LOCALIZATION OF WIRELESS SENSOR NETWORKS BASED ON PATH PLANNING OF MOBILE ROBOTS
}

\author{
Ebrahim Ashoori ${ }^{1}$, Iman Babagoli², Siavash Alipour ${ }^{3}$ \\ 1 Department of Electrical and Electronic Engineering, Malek-Ashtar University of Technology, Tehran, Iran, \\ e-mail: ebrahimashoori1383@gmail.com \\ 2 Department of Computer Engineering, Parsa University, Babolsar, Iran, e-mail: i.babagoli@gmail.com \\ 3 Department of Electrical and Electronic Engineering, Malek-Ashtar University of Technology, Tehran, Iran, \\ e-mail: siavash.alipur@gmail.com
}

Received: 2015.09.05

Accepted: 2015.11.14

Published: 2015.12.04

\begin{abstract}
With an explosive growth of wireless sensor networks (WSN), many of their features and applications have become important. Localization of sensor nodes is one of the most important problems in WSN whose accuracy has a very large impact on its performance. Global positioning system (GPS) is a well-known and powerful way which differentiates methods of its use on each node individually. But, because of high energy consuming and processing GPS, it is inappropriate for WSNs. Different algorithms are suggested to overcome the consumed cost of GPS by putting GPS on only some nodes instead of all nodes in the network for localization. So, for nodes localization, just a number of nodes exploit GPS and, they can help other nodes of network in localization via distribution of their coordinates. The use of a mobile robot to send signals to coordinate the target node localization is a good idea. The mobile robot should move in the right path and can localize node more accurately at lower cost. This paper proposes a new method to localize all nodes through some localized nodes based on graph theory in a tree and network topology. The proposed method provides better performance at the cost of accuracy and the number of nodes that can be made up of local consumption.
\end{abstract}

Keywords: sensor nodes, localization, global positioning system (GPS), wireless sensor network (WSN), graph theory.

\section{INTRODUCTION}

In many applications, the issue of localization in wireless sensor networks is among topics which play an important role. There are two main methods for nodes' localization. They include anchor-based methods and an anchor-free method. A significant number of localization methods use a number of nodes. These nodes know already their positions and play an important role in localization of other nodes. The positions of these nodes are determined by hand or by GPS. These nodes are called anchor nodes. As their name implies, anchor-free methods do not need any anchor and in these methods, the final place of sensor node will depend on the coordinates of map and the presence or absence of connectivity between different nodes is used for localization.

The advantage of anchor-based localization methods in comparison to the anchor-free one is that these methods consume less energy and there is no need to determine the domain of network deployment and the procedure of nodes distribution before. In this case, it is possible that we cannot use anchor-based nodes so we need to use anchor-free nodes. Because of lower cost and a decrease in the consumed energy in anchor-based methods, it is very important that just a few numbers of nodes shall be equipped with GPS and these nodes should send their co- 
ordinates to other sensors to help them in localization. Such methods have relatively low costs but will have higher precision in localization to the anchor-free methods.

Now, if we can perform localization helping a mobile robot, which is aware of its place (for example, using GPS), we can again decrease the consumed energy and cost. In this way, the planning of mobile robot is regarded as a basic and fundamental problem because the accuracy of localization is always influenced by robot movement path. In this paper, path planning of mobile robot and nodes localization is studied that uses the power of the received signal (RSSI). This method uses suggested algorithm by sichitiu and others to obtain the place of nodes from their travelled distance. In the rest of the paper, first we discuss previous works in assessing the distance and nodes localizations and the propose a brief description by sichitiu and others, then we study graph theory and spanning tree for path design of mobile robot and suggest travelled distance algorithm with the names of MMNF and LDF for spanning tree and these algorithms works better and less consuming than BRF and BTG. Finally, we discuss simulation scenarios and conclusions and finally we conclude the paper.

\section{LOCALIZATION WITH MOBILE ROBOTS}

There are two general methods for localization with mobile robots. The first class is the methods in which a mobile robot has not its local information, whereas the second class is methods in which a mobile robot has information on its local position. This local position information can be made available for a mobile robot by GPS.

\section{First class}

In different uses, it is not enough to know the relative localization of nodes and it needs to know the exact place of nodes. Different works are devoted to this method of localisation the use of GPS in their methods.

\section{Second class}

In methods in which a mobile robot does not have access to information on its local position, a mobile robot considers its beginning point as the coordinates of origin and moving along the sensor network domain, it sends its relative co- ordinates towards other sensors of the network. This method is usually used when we want to use localization information for routing in sensor network. The reason is that sensors' relative information is enough to determine the smallest distance and it does not need local information.

\section{Previous works}

Pathiran et. al [2] used radio waves and mobile robot for localization and measurement of the power of the received signal to estimate the distance of a mobile robot. Their simulation was made for four sensors and they did not present a clear path for movement of their mobile robot. Corke et. al $[3,4]$ used a flying robot which is equipped with GPS for localization and the measurement of radio waves' power to estimate the distance to a mobile robot. There are fixed robots in their method which localize a percentage of network nodes. Sichitiu and Ramadurai $[5,6]$ used GPS and measurement of the received signal power of radio waves to determine the distance between the node and mobile robot. $\mathrm{Su}$ et. al $[7,8]$ presented a method independent of the distance. In this method, a mobile robot which is equipped with GPS moves in operation environment and broadcasts its location. To design the path of mobile robot so that network nodes can localize the robot using the received signals of their own, three static paths of Scan, Doublescan and Hilbert are suggested in [9]. Then, some methods were proposed to improve movement path of robot to increase the accuracy of localization. In [10], two paths of Circles and S-curves were suggested. Also, in [11], two dynamic algorithms for the path designing were called BRF and BTG were proposed which have better performance with respect to the energy consumed by robot compared with static methods of path designing.

\section{A method for distance measurement}

As it was mentioned before, we use localization method proposed by Sitchtiv et al. [1] in which RSSI is used for distance measurement. We consider Regulation step to construct PDF table before position computing step. This table is stored on all nodes and maps each RSSI to probability distribution function (PDF). The received RSSI by each node is converted to the distance between two nodes based on probability distribution function (PDF). 


\section{The process of nodes' localization}

In the most available localization methods such as $[2,5,6,7,12-14]$ several distributive localization patterns were proposed which do not need to equip GPS on all nodes of network, and a mobile robot is used to send signals containing beacon. In this way, nodes of network can compute their local situation using transmitted beacons of a mobile robot. In the other words, a mobile robot distributes beacon packet when passes periodically through the network area in specific intervals. When each node receives beacon packet, it refers to PDF table to obtain its distance to the guidance nodes which distribute beacon packets. This process will be repeated for each beacon Packet which is received via the network node. When the node receives more than three beacon packets from the robot, it can calculate its coordinates according to the following relations:

$$
\begin{aligned}
& \mathrm{r} 1=\sqrt{(x-x 1)^{2}+(y-y 1)^{2}} \\
& \mathrm{r} 2=\sqrt{(x-x 2)^{2}+(y-y 2)^{2}} \\
& \mathrm{r} 3=\sqrt{(x-x 3)^{2}+(y-y 3)^{2}}
\end{aligned}
$$

In these equations $(x 1, y 1),(x 2, y 2)$ and $(x 3$, $y 3)$ are the coordinates of the first, second and third received beacons, respectively. $r 1, r 2$ and $r 3$ are the distances estimated by RSSI algorithm for each of these received beacons. As a result, the coordinate $(x, y)$ is estimated. This position estimation is followed by a percentage of error which is negligible to a significant extent.

\section{BEFORE PATH DESIGNING FOR MOBILE ROBOT}

In this section, we focus on designing a path of a mobile robot to improve it. In fact, if the robot can select a better path for its motion as much as possible, it will have a significant effect on cost reduction and the efficiency of nodes localization as well. Among the previous methods, dynamic BTG method presented in paper [11] has an advantage in efficiency, which leads to a decrease of energy consumption by robot in comparison with other methods. In [11] the, BRF algorithm has also been suggested to design the path of the mobile robot which has less efficiency than the BTG. In the BTG and the BRF, to travel path by mobile robot, nodes of network are considered as nodes of a graph, and mobile robot moves towards a node which has a higher number of neighboring nodes. The difference between these two algorithms turns to their travelling the graph. The BTG acts as indepth and the BRF acts as cross-sectional. In this paper, we propose two new dynamic algorithms called LDF and BRF to improve overhead cost which resulted from path designing of a mobile robot. Moreover, higher number of nodes can be localized by a mobile robot. The proposed LDF and BRF algorithms have been formed based on graph theory. The following assumptions have been considered to design the path of mobile robot by applying graph theory:

1. Nodes of wireless sensor networks use multidirectional antennas.

2. The communication area is circular-shaped and by radius of $R_{c}$. The communication radius of robot is also $R_{m}$. Also, for simplicity, we assume that these two radiuses are equal. Therefore, we use the abbreviation $R_{m}$ to express the length of communication radius.

3. All wireless communications between the nodes are bidirectional.

- Definition 1: Neighboring nodes - The node $V_{n}$ is called as neighboring-node of $V_{i}$ if during visiting time of robot with the node $V_{i}$, the distance between $V_{n}$ and robot be less than $r_{m}$.

- Definition 2: Internal neighboring nodes The node $V_{n}$ is called internal node of $V_{i}$ if the distance between $V_{n}$ and the robot be less than $2 r_{m} / 3$ when robot visit the node $V_{i}$.

- Definition 3: Marginal neighboring nodes The node $V_{n}$ is called marginal node of $V_{i}$ if during visiting time of robot with the node $V_{i}$, the distance between $V_{n}$ and the robot be less than $r_{m}$ and more than $2 r_{m} / 3$.

- Definition 4: Non-directional graph - In graph $G=(V(G), E(G)), V(G)$ and $E(G)$ are wireless sensor networks' nodes, and communication paths between nodes, respectively which are called as vertex and manes of nondirectional graph.

- Definition 5: Vertices weight is non-directional graph - if the number of unknown neighboring nodes $V_{i}$ is n, the weight of node $V_{i}$ is considered as $1 / n$.

- Definition 6: Spanning tree - spanning tree is a sub-tree which includes all vertices of graph $G$ and $I$ enough number of manes to construct the connected sub-graph but it has not any cycle. 


\section{PROPOSED ALGORITHM}

\section{LDF algorithm}

The proposed LDF algorithm is a very simple algorithm which acts based on the nearest distance to the neighboring nodes. In fact, this algorithm regards a marginal neighbor which has the least distance to the robot to select the next node which mobile robot moves towards it. The advantage of LDF algorithm is that by travelling shorter distances, we expect the nodes would be localized better because the distribution of localization signals by the robot will become higher and more effective. Meanwhile, since a greater number of signals will be distributed in the network by mobile robot, a greater number of network nodes can localized them. This is an important advantage for this algorithm which is able to localize a greater number of the network nodes work without overhead and energy wasting. By increasing precision and the quantity of nodes' localization, the consumed energy of robot will increase a well. This occurs due to a trade-off between localization precision and the consumed energy. It should be mentioned that the factor of the consumed energy in localization of mobile robot is fundamentally subjected to two factors of pathlength of mobile robot path, and the number of signal that the mobile robot sends for localization of network nodes. Here, we assume that the power of transmitted signal has a constant value. The pseudo-code of proposed LDF algorithm is expressed in algorithm 1 .

\section{MMNF algorithm}

The next proposed algorithm is MMNF algorithm. This algorithm considers the number of marginal neighbors of the robot to find the next node that the robot moves towards it. Selection of a node with the most marginal neighboring nodes leads to covering more space by robot in any step. In this way, smaller number of steps and consequently shorter distance will be traveled by robot in the network. As a result, the consumed energy will be saved significantly. The pseudo-code of the proposed MMNF algorithm has been shown in algorithm 2.

It should be noticed that choose any of the proposed methods is completely dependent on the conditions and topology of the network.

\section{SIMULATIONS}

The performance of the proposed algorithms (i.e. the order of visiting) in a constant conditions includes a number of nodes, network dimensions and radius of communication in the simulation environment is shown in Figures 1 and 2. Simulation environment is $35 \times 35 \mathrm{~m}$ in size, the number of nodes is 50 and communication radius is $10 \mathrm{~m}$.

In these figures, green-colored nodes represent network nodes and red-colored nodes represent the nodes visited by the mobile robot and movement robot path in any of the above-mentioned algorithms has been marked by red color. To compare between the proposed algorithms and the previous dynamic algorithms, several simulation scenarios are conducted which we refer to three cases of these scenarios and then we will discuss and analyze the contribution of the obtained results. In simulation scenarios, three criteria including the number of visited nodes, the travelled distance and the number of the localized nodes by the robot, have been examined against

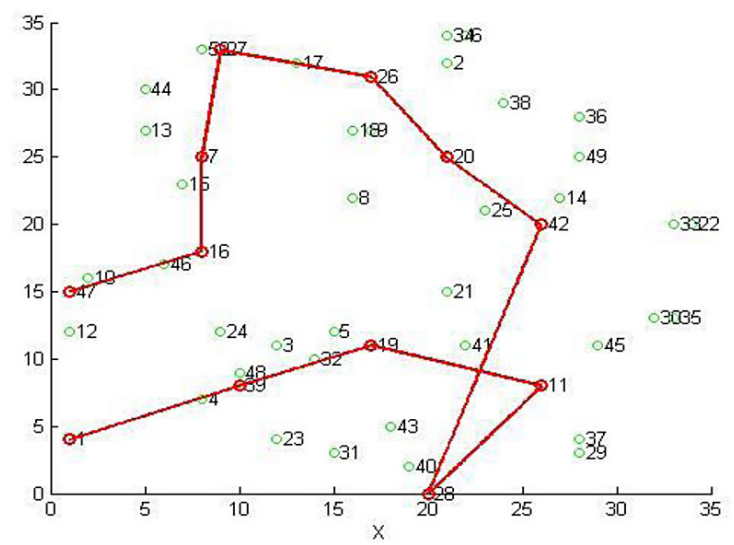

Fig. 1. The sequence of nodes visiting in MMNF algorithm

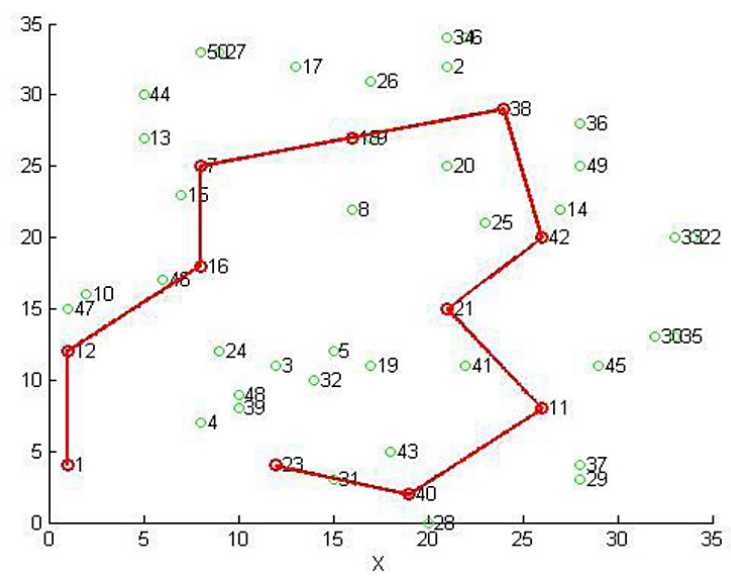

Fig. 2. The sequence of nodes visiting in LDF algorithm 
three factors including a change in the number of nodes, a change in simulation dimensions and a change in communication radius, respectively.

Scenario 1. Effect of the number of the visited nodes on the increase of simulation dimensions. In this scenario, we want to assess number of visited nodes against changes of simulation dimensions in a simulation environment with a specific number of nodes and along with a usual communication radius. Here, we changed the simulation dimensions from 100 to $500 \mathrm{~m}^{2}$. Also, we assumed that number of nodes as $300 \mathrm{~m}^{2}$ and communication radius $(\mathrm{rm})$ is $40 \mathrm{~m}$.

From Figure 3, it can be seen that the proposed MMNF algorithm in a usual simulation environment has a lower number of visited nodes in compared with BTG, BRF and the propose LDF algorithm. As we expect, it is normal that the number of the visited nodes in the proposed LDF algorithm is also more than BTG algorithm. Considering shorter distances causes that across a network a higher number of steps would be travelled. When network dimensions become extensive, due to the distribution of nodes in the environment, a mobile robot cannot find a neighbor in the BRF algorithm. As a result, it is stopped very rapidly quickly and covers little nodes. We regard two states for more detailed comparison between the algorithms under these conditions:

The first state is when the dimensions of the network circumferences are $100 \mathrm{~m}^{2}$. In this state, the numbers of visited nodes of MMNF, LDF, BTG and BRF algorithms are 8, 11, 8 and 13, respectively. In other words, it can be mentioned that the above algorithms should be visited by $2.66 \%$,
$3.66 \%, 2.66 \%$ and $4.33 \%$ of network nodes respectively, to cover all network nodes. In this state, MMNF shows the same performance with BTG. This is a single state in which such a problem is observed. Also, from the growth of MMNF in diagram, it can be found that when network dimensions increase, the performance of MMNF algorithm has a significant performance compared with other algorithms. Second state is when circumference dimension is $500 \mathrm{~m}^{2}$. In this state, visited nodes of MMNF, LDF, BTG and BRF algorithms are $86,115,113$ and 6 , respectively. In other words, it can be said that the algorithms for covering network nodes should visit $28.66 \%, 38.33 \%$, $37.66 \%$ and $2 \%$ of network nodes, respectively Note that the BRF does not cover network nodes. In this state, the improvement of the MMNF algorithm compared with the BTG and the LDF is 9\% and $9.66 \%$, respectively. These values show absolute superiority of MMNF algorithm in this case. Also, they show that the performance of LDF algorithm approaches to the BTG when dimensions of network circumference increase.

Scenario 2. Effect of distance travelled by the robot on the increase of communication radius. In this scenario we want to assess the travelled distance against changes of communication radius with a number of constant and same-sized nodes of a usual network. For this purpose, we change the communication radius of mobile robot from $20 \mathrm{~m}$ to $55 \mathrm{~m}$, while the number of network nodes is constant and equal to 500. Also, the dimension of network circumference is $250 \mathrm{~m}^{2}$.

According to Figure 4, all algorithms have a descending motion in this state. It means they

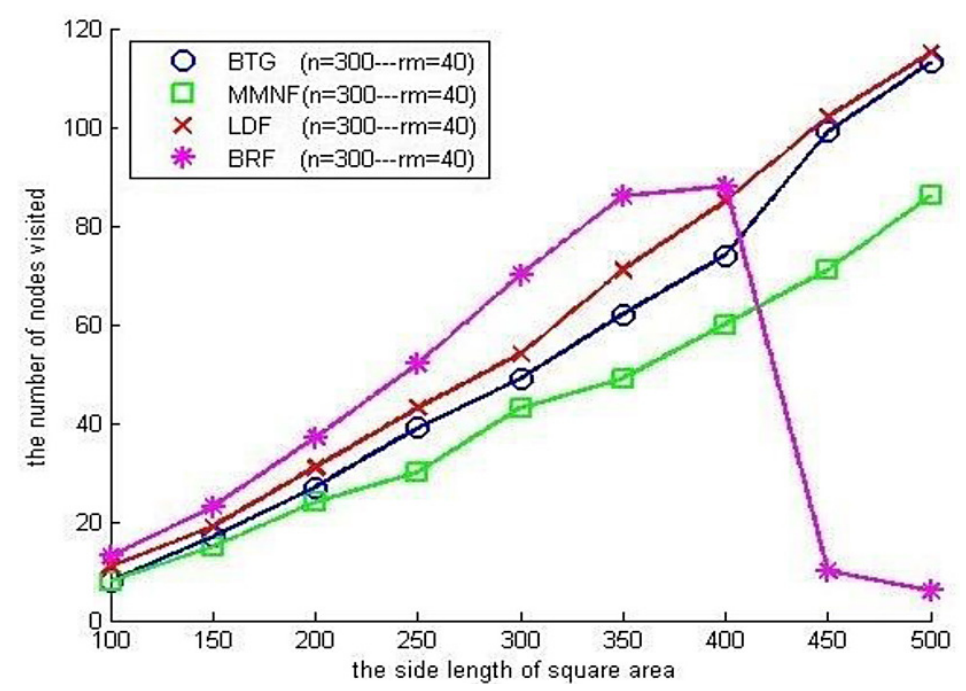

Fig. 3. The number of the visited nodes against increase in environment dimensions 


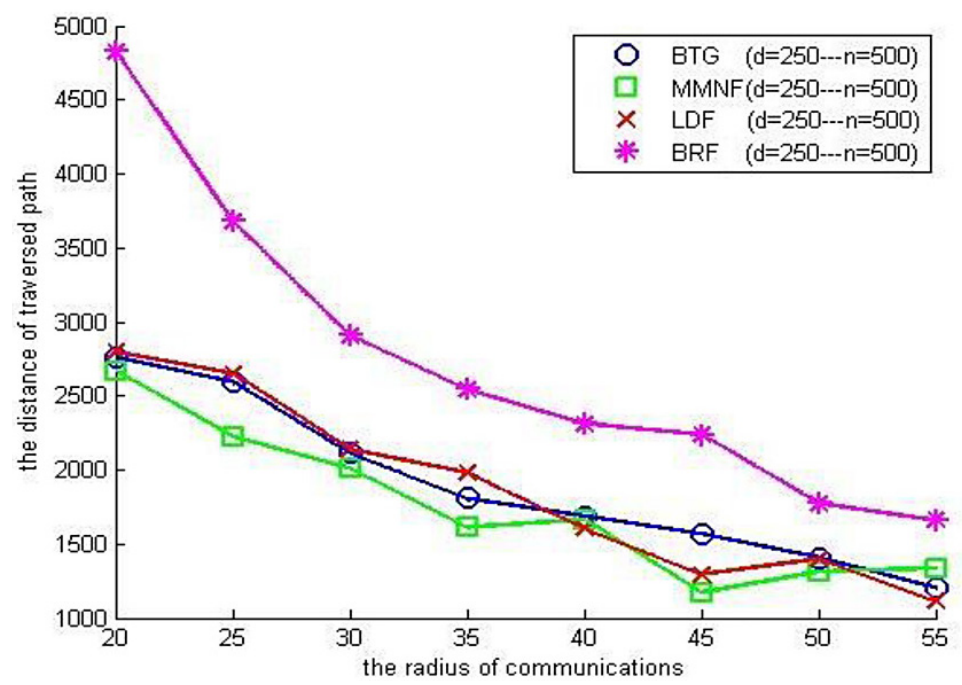

Fig. 4. The distance travelled by robot against increase in communication radius

travel a shorter distance which leads lower energy consumption while communication radius increases. In general, it seems that despite close performance of algorithms, MMNF algorithm acts better than other algorithms. For more precise study, we examine three states: when communication radius of the robot is 1) $20 \mathrm{~m}, 2) 40$ $\mathrm{m}$ and 3) $55 \mathrm{~m}$.

In the first case, distance length of MMNF, LDF, BTG and BRF algorithms are about 2669, 2797, 2761 and $4827 \mathrm{~m}$, respectively. Their percentage equivalent in compared with circumference area of $4.27 \%, 4.48 \%, 4.42 \%$ and $7.72 \%$, respectively. In radius $40 \mathrm{~m}$, these values are 1665 , 1689,1603 and $2312 \mathrm{~m}$, respectively which are equivalent to $2.66 \%, 2.56 \% 2.70 \%$ and $3.7 \%$, respectively. Finally, in dimensions $500 \times 500$, these values are $2.14 \%, 1.78 \%, 1.92 \%$ and $2.65 \%$, respectively. In this way, we cannot certainty com- pare superiority of each algorithm to others. But, we can generally say that the proposed algorithms have perfectly better performances, compared to the previous algorithms.

Scenario 3. Effect of localized nodes number on increase of the number of network nodes. In this scenario, we want to assess the localized nodes number against increase of the number of network nodes on a simulation environment with constant dimensions along with a suitable communication radius. For this purpose, we change the number of network nodes from 250 to 600 while the dimensions of simulation environment are assumed 350 $\mathrm{m}^{2}$. Also, communication radius is $40 \mathrm{~m}$.

From Figure 5, we can see that except of the MMNF algorithm, there is no high difference between three other algorithms in respect of localized nodes number. However, the proposed LDF

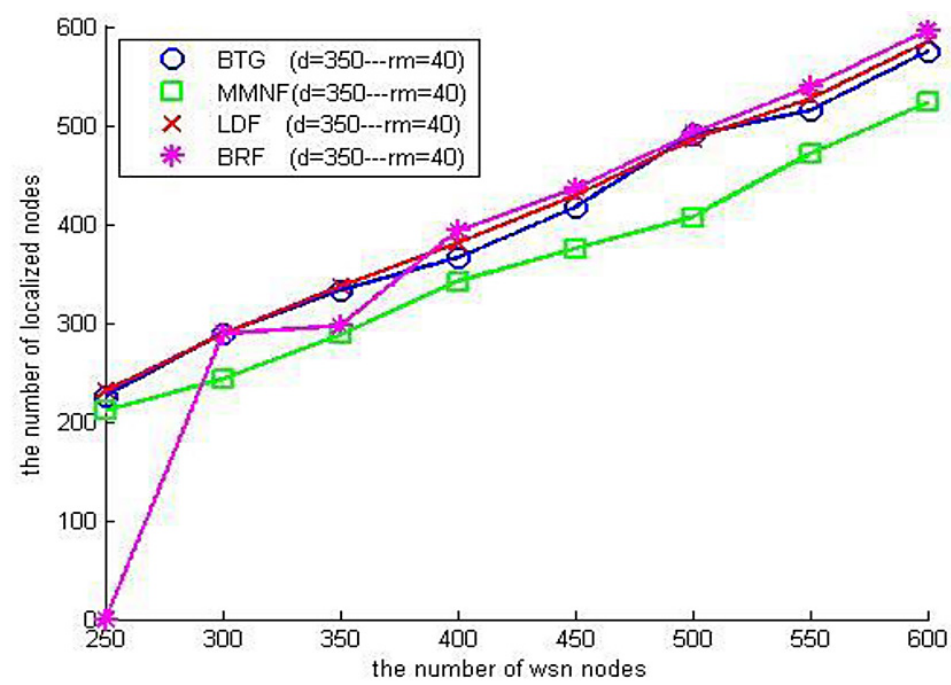

Fig. 5. The number of localized nodes against increase in the number of network nodes 


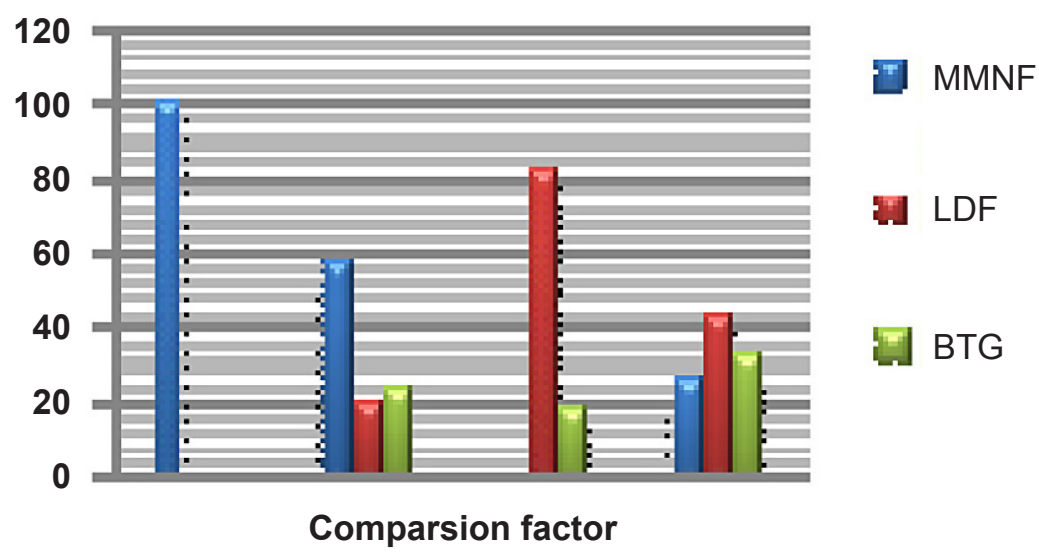

Fig. 6. The diagram for the percentage of superiority times of algorithms

algorithm exhibit very desired performance compared with other two algorithms. Figure 6 shows superiority percentage of any algorithm in 100 times of simulation with the same conditions. In this diagram, the parameter of precision has been involved which shows that LDF algorithm can improve precision of localization. In fact, if localized nodes number has fewer errors, localization precision will be increased. From the diagram in Figure 6, we can see that LDF algorithm is more precise than other algorithms.

\section{CONCLUSIONS}

Application of wireless sensor networks causes that localization can be considered as one of the most important task in this field. Use of mobile robot is one suitable way which tries to decrease the cost of localization and make it more effective for sensor network nodes. Path travelling algorithms for the mobile robot are divided to two static and dynamic such that dynamic algorithms are more effective for dynamic networks. This paper proposes two dynamic algorithms named LDF and MMNF for robot path travelling which have better performance in comparison with previous dynamic algorithms according to consuming energy and localization precision terms. Efficiency of each proposed algorithm is subjected to goals that we have from a mobile robot. If the consuming energy in robot is important, the best method is the MMNF algorithm. On the other hand, if we want to localize more number of nodes, and also precision is important goal for localization, the LDF is suggested. From the statistics which was obtained by simulation, in $60 \%$ of cases, MMNF has a better efficiency in respect of the traverse distance to the other algorithms.
In $80 \%$ of cases, the LDF algorithm has better efficiency in respect of localized nodes. In $40 \%$ of cases, LDF algorithm has better efficiency than other algorithms.

\section{REFERENCES}

1. Koutsonikolas D., Das S.M., Hu Y.C. Path planningof mobile landmarks for localization in wireless sensor networks. In: Proceedings of ICDCSW, 2006.

2. Corke P., Peterson R., Rus D. Localization and navigation assisted by cooperating networked sensors and robots. International Journal of Robotics Research, 24(9), 2009, 771-786.

3. O'Hara K.J. and Balch T.R. Distributed path planning forrobots in dynamic environments using a pervasive embedded network. In: Third International Joint Conference on Autonomous Agents and Multiagent Systems (AAMAS '04), Vol. 3, 2004, 1538-1539.

4. Cheng X., Thaeler D.C.A., Xue G. TPS: A timebased positioning scheme for outdoor wireless sensor networks. In: Proceedings of IEEE Infocom, March 2008.

5. Corke P., Peterson R., Rus D. Networked robots: Flying robotnavigation using a sensor net. In: Proceedings of ISRR, October 2003.

6. Krishnamachari G.B., Lerman K., Pattem S. Distributed online localization in sensor networks using a moving target. In: Proceedings of IPSN, April 2004.

7. Sichitiu M.L., Ramadurai V. Localization of wireless sensor networks with a mobile beacon. In: Proceedins of MASS, September 2004.

8. Doherty L.X., Pister Y.S.J., Ghaoui L.E. Convex position estimation inwireless sensor networks. In: Proceedings of IEEE Infzczm '01, Anchorage, AY, USA, 2005. 
9. Hu L. and Evans D. Localization for mobile sensor networks. In: Proceedings of ACM MobiCom, September 2004.

10. Ji X. and Zha H. Sensor positioning in wireless ad-hoc sensor networks using multidimensional scaling. In: Proceedings of IEEE Infocom, March 2009.

11. Krishnan P., Krishnakumar A.S., Ju W.-H., Mallows C., Ganu S.. Asystem for LEASE: System for location estimation assisted by stationaryemmiters for indoor RF wireless networks. In: Proceedings of IEEE Infocom, March 2004.

12. Pathirana N., Bulusu N., Savkin A.V., Jha S. Node localization using mobile robots in delay-tolerant sensor networks. IEEE Transactions on Mobile Computing, 4, 2004, 285-296.

13. Kuriakose J., Joshi S., Vikram Raju R., Kilaru A. A review on localization in wireless sensor networks. Advances in Intelligent Systems and Computing. 264, 2014, 599-610.

14. Jo K., Chu K., Kim J., Sunwoo M. Distributed vehicle state estimation system using information fusion of GPS and in-vehicle sensors for vehicle localization. In: Proceedings of Intelligent Transportation Systems (ITSC). 14 ${ }^{\text {th }}$ International IEEE Conference on, 2011, 2009-2014, 